\title{
Effects of Inorganic Anions on the Photopolymerization of Acrylamide Sensitized by 2,3-Butanedione in Aqueous Solutions
}

\author{
Fukuo TAKEMURA, Kaoru IWAI, Minako IKESU, \\ Kimi ITOH, and Atsuko MATOIKE \\ Department of Chemistry, Faculty of Science, \\ Nara Women's University, Nara 630, Japan
}

(Received December 21, 1987)

\begin{abstract}
In aqueous solutions, 2,3-butanedione (biacetyl: BA) served as a sensitizer for the photopolymerization of acrylamide (AA). The presence of inorganic salts such as $\mathrm{KSCN}, \mathrm{KSeCN}$, and $\mathrm{NaN}_{3}$ increased not only the rate of polymerization but also the molecular weight of the polymer. On the other hand, $\mathrm{Na}_{2} \mathrm{SeO}_{3}$ increased the rate of polymerization markedly with apparently normal kinetic behavior. In the absence of any salt, the triplet excited state of BA was shown to act as a degradative chain transfer agent as well as a photo-sensitizer. The mechanism of this characteristic photosensitized polymerization is discussed.

KEY WORDS Photopolymerization / Sensitizer / Acrylamide / 2,3-

Butanedione / Biacetyl / Triplet Excited State / Inorganic Anion /

Degradative Chain Transfer /
\end{abstract}

The photopolymerization of vinyl monomers sensitized by organic carbonyl compounds has been investigated by many workers. ${ }^{1}$ Most of these studies were carried out in organic solvents. 2,3-Butanedione (Biacetyl: BA), an $\alpha$-diketone soluble both in water and organic media, was used as an efficient sensitizer for the polymerizations of monomers such as styrene and methyl methacrylate. ${ }^{2-5}$ But there has been little study on BA-sensitized polymerization in an aqueous medium. Recently, Encina and his coworkers ${ }^{6}$ reported that in the polymerization of $\mathrm{N}$-vinyl-2pyrrolidone and vinyl acetate, BA was a poor initiator and relatively high photoinitiation efficiencies were achieved only in the presence of methanol or water, by which BA was solvated giving a hydroxy-substituted alkanone effective at $300 \mathrm{~nm}$ irradiation.

We studied the photopolymerizations of vinyl monomers such as acrylonitrile, styrene, methyl methacrylate, and acrylamide (AA) sensitized by organic dyes ${ }^{7}$ and metal complexes. ${ }^{8-14}$ In a previous paper, ${ }^{13}$ we reported the photopolymerization of $\mathrm{AA}$ sensitized by the $\left[\mathrm{Co}\left(\mathrm{NH}_{3}\right)_{6}\right] \mathrm{Cl}_{3}$ or $\left[\mathrm{Co}(\mathrm{en})_{3}\right] \mathrm{Cl}_{3}$ (en: ethylenediamine)-KSCN system in aqueous medium. These metal complexes alone could not initiate the photopolymerization of $\mathrm{AA}$, but could sensitize the polymerization markedly in the presence of KSCN.

We thus studied such effects of inorganic salts on the photopolymerizations in aqueous solutions. BA is soluble in water and has a relatively unique property of exhibiting phosphorescence as well as fluorescence in a fluid solution at room temperature. Then, the effects of inorganic salts on the photopolymerization of AA sensitized by BA were studied kinetically and spectroscopically.

Contrary to the normal kinetic behavior of radical polymerization, ${ }^{15}$ the addition of some 
salts was found to increase not only the rates of polymerization but also the molecular weights of the polymer obtained. The mechanism of the polymerization is discussed in detail to account for this anormalous behavior.

\section{EXPERIMENTAL}

\section{Materials}

Acrylamide (AA) was recrystallized twice from benzene. 2,3-Butanedione (biacetyl: BA) was purified by vacuum distillation before use. Inorganic salts were of reagent grade and used without further purification.

\section{Experimental Procedures}

A freshly prepared sample solution was degassed by four freeze-pump-thaw cycles on a vacuum line, and then sealed into a standard 1$\mathrm{cm}$ spectrophotometer quartz cell (capacity, $4 \mathrm{ml})$.

The photopolymerization was carried out under irradiation at $30^{\circ} \mathrm{C}$. As a stationary light source, $500 \mathrm{~W}$ ultra-high pressure mercury lamp (Ushio USH-500D) was used together with a cutoff glass filter (Toshiba UV-D2 85\% transmittance at $365 \mathrm{~nm}$ ). The polymers were isolated by precipitation with methanol.

The rate of polymerization was determined from the weight of the polymer obtained in the reaction time, since the conversion almost linearly increased with time. The molecular weight of the polymer was measured by GPC calibrated by poly(ethylene oxide) in water or by an Ostward viscosimeter using the following equation, $[\eta]=6.8 \times 10^{-4} \bar{M}_{n}{ }^{0.66} \cdot{ }^{16}$

\section{Measurements}

Absorption spectra were measured with a Hitachi model 124 spectrophotometer. Emission spectra were recorded with a Hitachi model EPS-2 spectrophotometer $\left(\lambda_{\mathrm{ex}}, 365 \mathrm{~nm}\right)$. Since BA-phosphorescence was observable only in an oxygen-free solution at room temperature, sample solutions were deaerated in sealed cells. The flash photolysis apparatus was the same as described previously. ${ }^{13}$ The light intensities were measured with potassium tris(oxalato)ferrate(III) actinometer ${ }^{17}$.

\section{RESULTS AND DISCUSSION}

Photopolymerization of AA Sensitized by BA and Effects of Inorganic Salt Additives

Using BA as a sensitizer in aqueous solutions, both the rates of photopolymerization $\left(R_{\mathrm{p}}\right)$ of $\mathrm{AA}$ and molecular weights of the polymers obtained were nearly proportional to the concentrations of the monomer over the range of 0.3 to $1.5 \mathrm{M}$ at a given concentration of BA $(0.1 \mathrm{M})$.

As shown in Figure $1, R_{\mathrm{p}}$ was linear to the square root of the absorbed light intensity. This observation appeared in agreement with that for the normal radical polymerization terminated bimolecularly.

Under the same conditions, the presence of potassium thiocyanate $([\mathrm{KSCN}]=0.01 \mathrm{M})$ brought about an increase of the rate by a factor of about 4 (Figure 1). The effects of

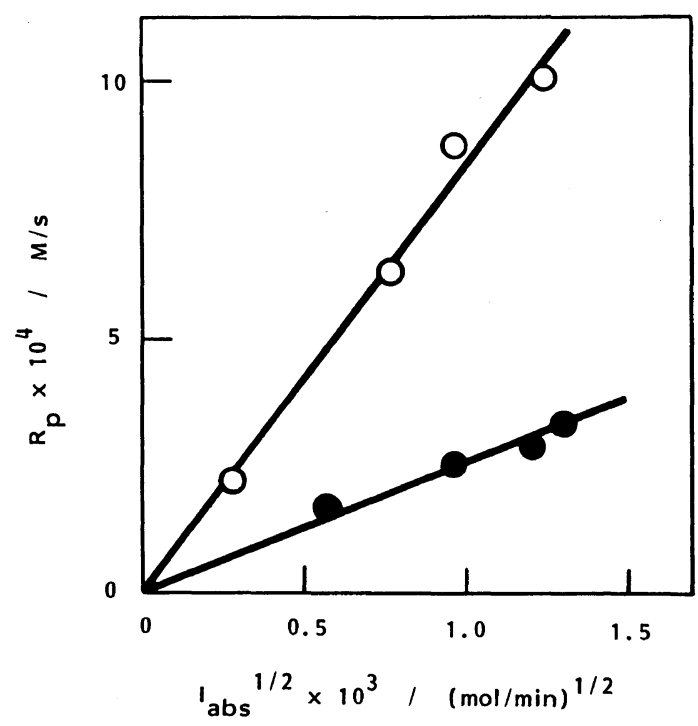

Figure 1. Dependence of the rate of photopolymerization on the square root of the absorbed light intensity in the absence $(O)$ and presence $(O)$ of $\mathrm{KSCN}:[\mathrm{AA}]=$ $1 \mathrm{moll}^{-1},[\mathrm{BA}]=0.1 \mathrm{moll}^{-1}$, and $[\mathrm{KSCN}]=0.01 \mathrm{moll}^{-1}$. 
various inorganic salts such as $\mathrm{NaN}_{3}, \mathrm{KSeCN}$, and $\mathrm{Na}_{2} \mathrm{SeO}_{3}$ as well as $\mathrm{KSCN}$ are shown in Figure 2, in which $R_{\mathrm{p}}$ is plotted against the square root of salt concentration. With respect to $\mathrm{KSCN}$, a linear relation between $R_{\mathrm{p}}$ and $[\mathrm{KSCN}]^{1 / 2}$ was observed throughout a concentration of the salt $\left(\mathrm{ca} .1 \times 10^{-4} \mathrm{M}\right)$, above which $R_{\mathrm{p}}$ was held almost constant and the

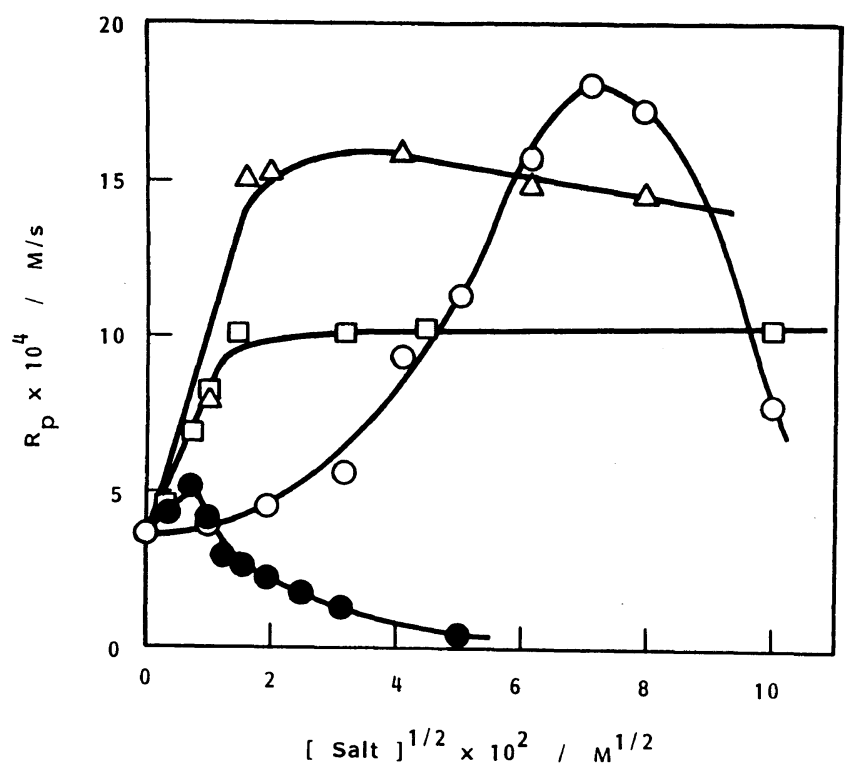

Figure 2. Dependence of the rate of photopolymerization on the square root of the concentration of the added salts: $(\square), \mathrm{KSCN} ;(\triangle), \mathrm{NaN}_{3} ;(\bigcirc), \mathrm{KSeCN} ;(\bigcirc), \mathrm{Na}_{2} \mathrm{SeO}_{3} ;[\mathrm{AA}]=1 \mathrm{moll}^{-1}$ and $[\mathrm{BA}]=$ $0.1 \mathrm{moll}^{-1}$

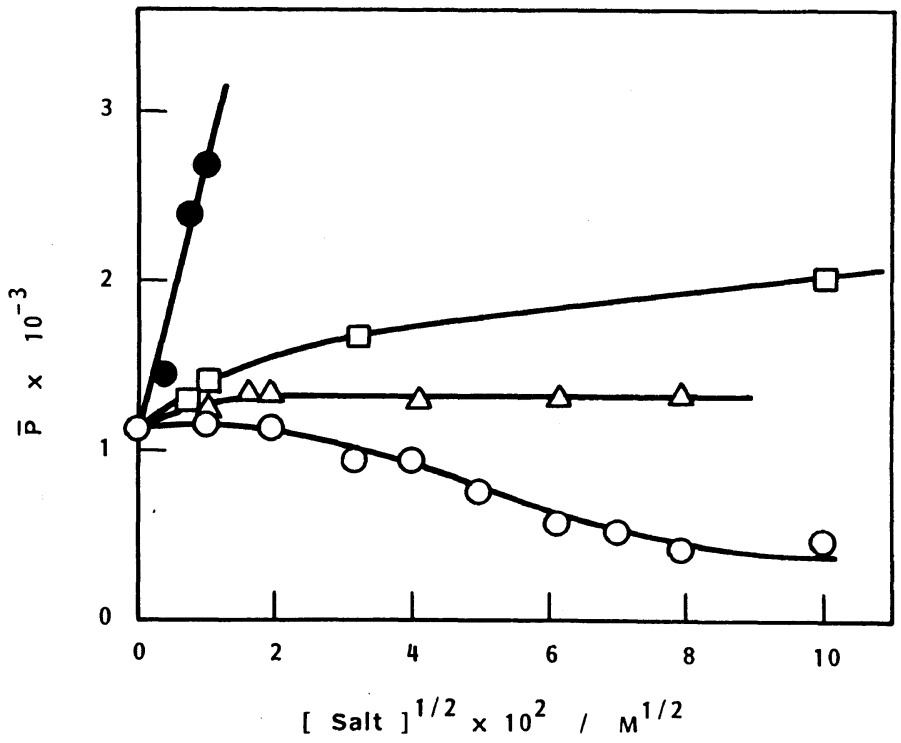

Figure 3. Dependence of the degree of polymerization $(\bar{P})$ on the square root of the concentration of the added salts: $(\square), \mathrm{KSCN} ;(\triangle), \mathrm{NaN}_{3} ;(\bigcirc), \mathrm{KSeCN} ;(\bigcirc), \mathrm{Na}_{2} \mathrm{SeO}_{3} ;[\mathrm{AA}]=1 \mathrm{moll}^{-1}$ and $[\mathrm{BA}]=$ $0.1 \mathrm{moll}^{-1}$ 
phosphorescence of BA was quenched completely. The observation with $\mathrm{NaN}_{3}$ was similar to that with $\mathrm{KSCN}$. With $\mathrm{KSeCN}, R_{\mathrm{p}}$ increased at lower concentrations, although $R_{\mathrm{p}}$ decreased considerably at high concentrations. In the presence of $\mathrm{Na}_{2} \mathrm{SeO}_{3}$, on the other hand, $R_{\mathrm{p}}$ increased gradually but progressively with increasing concentration of the salt, and then decreased at very high concentrations where BA might be squeezed out by salting-out.

In Figure 3, the degree of polymerization (DP) of the polymers obtained in the above experiments is plotted against the same abscissa as that in Figure 2. It is well known that in the normal radical polymerization of a monomer, the molecular weight of the polymer obtained decreases with increasing $R_{\mathrm{p}}$ at a constant concentration of the monomer because of faster bimolecular radical termination. Nevertheless, the salt additives tested except $\mathrm{Na}_{2} \mathrm{SeO}_{3}$ enlarged the molecular weights of polymers along with increasing $R_{\mathrm{p}}$. These observations have not been reported as far as we know. On the other hand, the polymers obtained in the presence of $\mathrm{Na}_{2} \mathrm{SeO}_{3}$ became shortened gradually with increasing concentration of the salt. This behavior is apparently of a well-known type.

\section{Relation between $R_{\mathrm{p}}$ and $D P$ of the Polymer}

As is well known, almost all examples of photopolymerization are of free-radical character and $R_{\mathrm{p}}$ can be expressed as

$$
R_{\mathrm{p}}=k_{\mathrm{p}}\left(\frac{2 \times 2.3 \phi \varepsilon \mathrm{d} I_{0}}{k_{\mathrm{t}}}\right)^{1 / 2}[\mathrm{BA}]^{1 / 2}[\mathrm{M}]
$$

and DP for the case of no chain transfer is

$$
\mathrm{DP}=\frac{k_{\mathrm{p}}[\mathrm{M}]}{\left(2 \times 2.3 \phi \varepsilon \mathrm{d} I_{0} k_{\mathrm{t}}\right)^{1 / 2}[\mathrm{BA}]^{1 / 2}}=\frac{\left(k_{\mathrm{p}}[\mathrm{M}]\right)^{2}}{k_{\mathrm{t}} R_{\mathrm{p}}}
$$

where notations for the rate constants of propagation and termination and for the concentration of monomer are the same as usual, and $\phi, \varepsilon, d$, and $I_{0}$ are the quantum yield, extinction coefficient, light-pass length in $\mathrm{cm}$, and light intensity, respectively. Then, at a

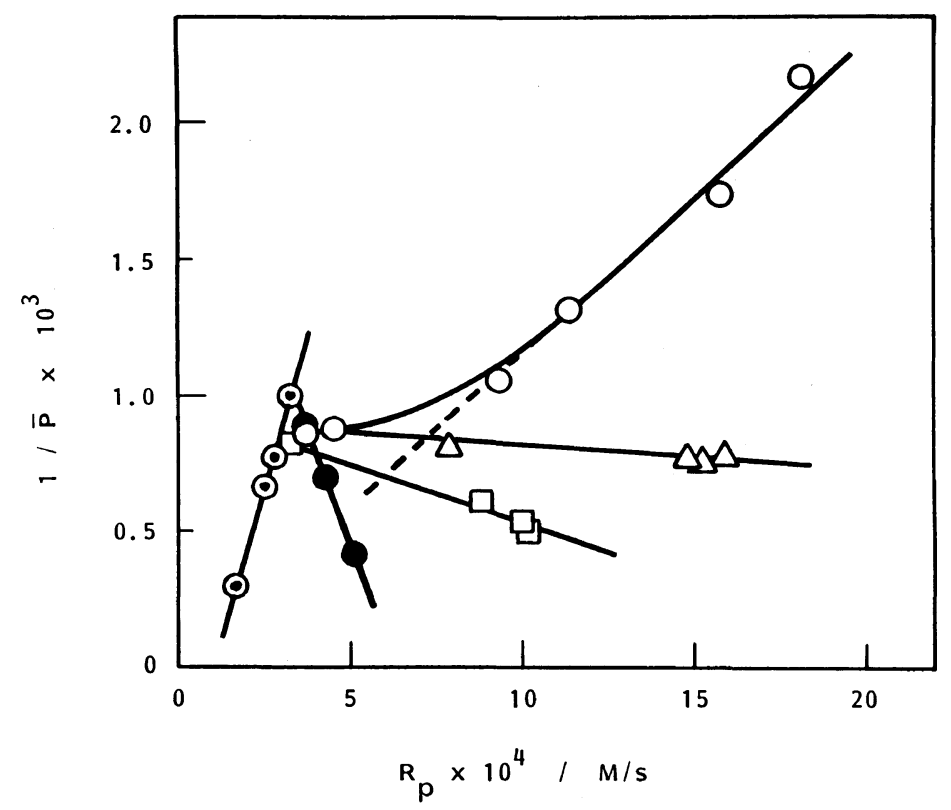

Figure 4. Plots of the reciprocal of the degree of polymerization $(1 / \bar{P})$ against the rate of polymerization: $(\square), \mathrm{KSCN} ;(\triangle), \mathrm{NaN}_{3} ;(\bigcirc), \mathrm{KSeCN} ;(\bigcirc), \mathrm{Na}_{2} \mathrm{SeO}_{3} ;(\odot)$, Non. 
stationary light intensity and constant concentration of monomer, $R_{\mathrm{p}}$ should be proportional to the square root of the concentration of sensitizer and DP inversely proportional to $R_{\mathrm{p}}$.

In Figure 4 , the reciprocals of DP are plotted against $R_{\mathrm{p}}$ in the absence and presence of various inorganic salts. Some interesting features are seen in the figure.

1) The line for the absence of any salt, on which the concentration of BA is over the range of 0.001 to $0.1 \mathrm{M}$, has a steep positive slope. Further, when the light intensity was lowered through a neutral density filter at $0.1 \mathrm{M} \mathrm{BA}$, the plot of $1 / \mathrm{DP}$ against $R_{\mathrm{p}}$ was almost on the same line as shown above. Therefore, the excited state of BA may be responsible for this behavior.

2) In the presence of $\mathrm{Na}_{2} \mathrm{SeO}_{3}$, the line was of moderate positive slope in higher rate region but the slope became gentle in relatively low rate region. The extrapolation of the linear part of the plots passed through the original point of axes, indicating a normal behavior of radical polymerization in that region.
3) For cases in the presence of $\mathrm{NaN}_{3}$, $\mathrm{KSCN}$, and $\mathrm{KSeCN}$, the lines had more negative slopes in this order, that is, DP also increased with increasing $R_{\mathrm{p}}$ in contrast to usual cases.

4) The molecular weight of the polymer, obtained at a given concentration of BA $(0.1 \mathrm{M})$ in the absence of any salt, was significantly small in spite of the low rate of polymerization. This shows that BA also may act as a retarder in the process although it played a role in sensitization on the other hand. Therefore, the addition of salts seems to weaken the retarding effect of BA.

In the absence and presence of KSCN, $R_{\mathrm{p}}$ is plotted against the square root of the concentration of BA in Figure 5. It was shown that at higher concentrations of BA, the polymerization was suppressed because of degradative chain transfer by BA, but the regarding effect was removed by the presence of $\mathrm{KSCN}$ $(0.01 \mathrm{M})$.

Thermal polymerization of AA initiated by azobisisobutylonitrile (AIBN) in aqueous solution was found to be significantly retarded

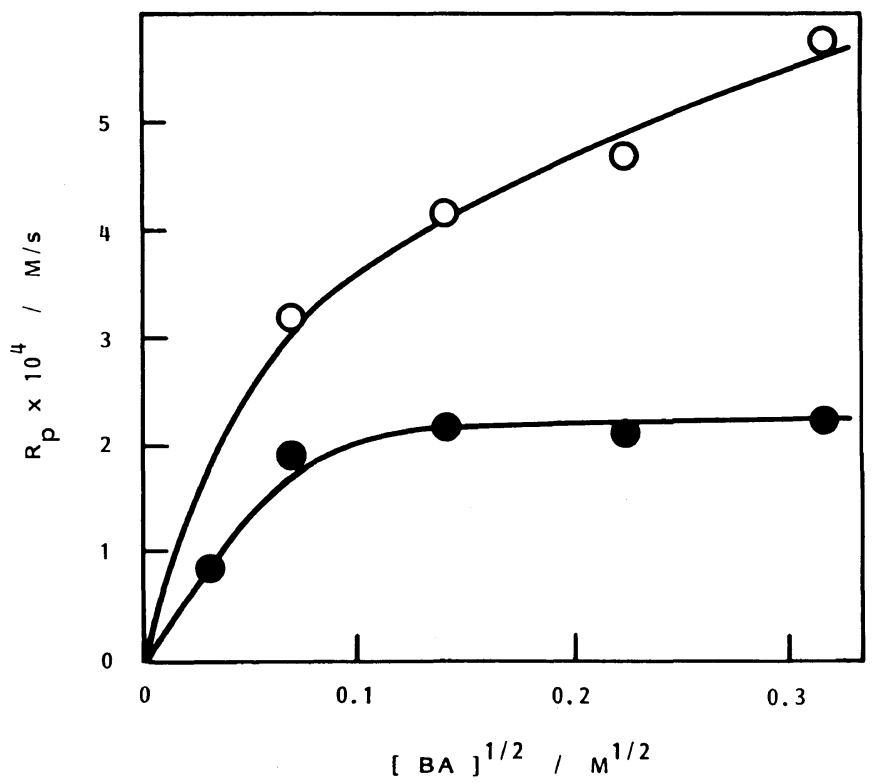

Figure 5. Plots of the rate of polymerization against the square root of the concentration of 2,3butanedione in the absence $(\bigcirc)$ and presence $(\bigcirc)$ of $\mathrm{KSCN}$ : $[\mathrm{AA}]=1 \mathrm{moll}^{-1}$ and $[\mathrm{KSCN}]=0.01 \mathrm{~mol}^{-1}$. 
by a small amount of BA in the dark as shown in Table I. This finding also shows BA itself to be a retarder of the polymerization of $\mathrm{AA}$ in aqueous solution.

\section{Quenching of BA Phosphorescence by Inorganic \\ Anions}

BA is one of a few examples which exhibit phosphorescence in a fluid solution at room temperature. On irradiation $(\lambda=365 \mathrm{~nm}) \mathrm{BA}$ undergoes $n \rightarrow \pi^{*}$ transition to give the corresponding singlet exited state, from which the triplet state is formed with a quantum yield of nearly unity. As the triplet lifetime $(0.145 \mathrm{~ms})$ is about $2 \times 10^{4}$ times greater than the singlet lifetime (6.3 ns), ${ }^{18}$ the concentrations of anions

Table I. Effects of biacetyl on the AIBN-initiated thermal polymerization of acrylamide ${ }^{a}$

\begin{tabular}{lc}
\hline Initiator & $R_{\mathrm{p}} \times 10^{5} / \mathrm{M} \mathrm{s}^{-1}$ \\
\hline $\mathrm{AIBN}^{\mathrm{b}}$ & 29.8 \\
$\mathrm{AIBN}^{\mathrm{b}}+\mathrm{BA}^{\mathrm{c}}$ & 1.95 \\
$\mathrm{BA}^{\mathrm{c}}$ & 1.71 \\
\hline
\end{tabular}

a $[\mathrm{AA}]=1.0 \mathrm{M}$ in aqueous solution at $60^{\circ} \mathrm{C}$.

b AIBN was saturated in the solution.

c $[\mathrm{BA}]=0.1 \mathrm{M}$. which efficiently quench phosphorescence leave fluorescence unaffected.

To study the interactions between the excited BA and inorganic anions, the intensities of BA-phosphorescence at $512 \mathrm{~nm}$ were measured in the presence of these salts and are plotted against their concentrations in Figure 6 (Stern-Volmer plot). The triplet excited state was quenched by $\mathrm{SeCN}^{-}, \mathrm{SCN}^{-}$, and $\mathrm{N}_{3}{ }^{-}$, but not by $\mathrm{SeO}_{3}{ }^{2-}$. From the values of the quenching rate constant $k_{\mathrm{q}}$ in Table II, calculated from $K_{\mathrm{Sv}}$ and the triplet lifetime $(0.145 \mathrm{~ms})$, the behavior of the anions except $\mathrm{SeO}_{3}{ }^{2-}$ appears to be correlated with their redox potentials, indicating that a charge

Table II. Stern-Volmer constants $\left(K_{\text {sv }}\right)$ and quenching constants $\left(k_{\mathrm{q}}\right)$ for quenching of biacetyl phosphorescence by some inorganic anions

\begin{tabular}{|c|c|c|c|}
\hline \multirow{2}{*}{ Anions } & $K_{\mathrm{Sv}}$ & $k_{\mathrm{q}}$ & Redox potential \\
\hline & $\mathbf{M}^{-1}$ & $M^{-1} s^{-1}$ & V \\
\hline $\mathrm{SeCN}^{-}$ & $8.65 \times 10^{5}$ & $6.0 \times 10^{9}$ & 0.42 \\
\hline $\mathrm{SCN}^{-}$ & $3.18 \times 10^{5}$ & $2.2 \times 10^{9}$ & 0.77 \\
\hline $\mathrm{N}_{3}^{-}$ & $5.10 \times 10^{4}$ & $3.5 \times 10^{8}$ & $1.33^{\mathrm{a}}$ \\
\hline $\mathrm{SeO}_{3}{ }^{2-}$ & $2.4 \times 10^{2}$ & $1.7 \times 10^{6}$ & 0.03 \\
\hline
\end{tabular}

${ }^{\text {a }}$ For the $\mathrm{N}_{3} \cdot / \mathrm{N}_{3}{ }^{-}$redox couple. ${ }^{21}$

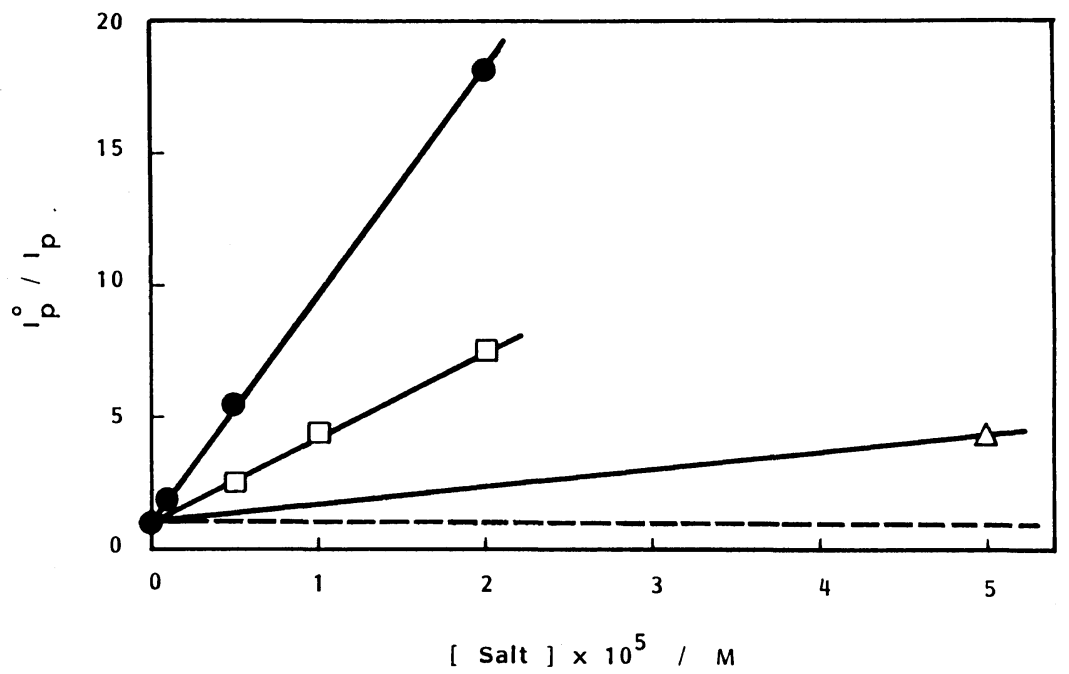

Figure 6. Plots of the intensity ratio of the phosphorescence of 2,3-butanedione $\left(I_{\mathrm{p}}{ }^{\circ} / I_{\mathrm{p}}\right)$ at $512 \mathrm{~nm}$ against the concentration of the added salts: $(\square), \mathrm{KSCN} ;(\triangle), \mathrm{NaN}_{3} ;(\mathbf{O}), \mathrm{KSeCN} ;----, \mathrm{Na}_{2} \mathrm{SeO}_{3}$ (observed point is out of range); $[\mathrm{BA}]=0.1 \mathrm{moll}^{-1}$. 
transfer process from the anion to the triplet of $\mathrm{BA}$ is responsible for quenching. But the lack of quenching effect of $\mathrm{SeO}_{3}{ }^{2-}$ in spite of its high reducing power shows that no direct interaction of the ion with the triplet state actually occurs.

In a detailed study on quenching of triplet states by inorganic ions, Treinin and Hayon ${ }^{19}$ found that the triplet states of acetonaphthone and acetone were quenched less effectively by $\mathrm{SO}_{3}{ }^{2-}$ than by $\mathrm{I}^{-}$in spite of the stronger reducing power of $\mathrm{SO}_{3}{ }^{2-}$. Thus, in analogy with $\mathrm{SO}_{3}{ }^{2-}$ the triplet of $\mathrm{BA}$ might be hardly quenched by $\mathrm{SeO}_{3}{ }^{2-}$, and then the accelerating effect of the salt for the polymerization would be ascribed to the regeneration of initiating radicals from the retarding products by means of the salt.

\section{BA-Sensitized Formation of Inorganic Anion Radicals by Flash Photolysis}

The quantum yield of intercarbonyl $\mathrm{C}-\mathrm{C}$ bond breakage during the photolysis of BA solution is known to be very low (0.01) as compared to that of the triplet formation ( $c a$. 1). ${ }^{20}$ Then, in the presence of sufficient high concentration of $\mathrm{KSCN}(0.01 \mathrm{M})$ to quench the triplet, flash photolysis of BA solution was found to give a transient radical anion $(\mathrm{SCN})_{2} \cdot{ }^{-}$which had an absorption maximum at $475 \mathrm{~nm}$ and half-life period of about $60 \mu \mathrm{s}$. The half-life period of the radical anion observed in this experiment was about one-third that reported previously, ${ }^{12}$ suggesting that the radical anion might interact with a ground state of BA. Moreover, the addition of AA monomer into the system resulted in increased decay rate of the transient with increasing concentration of the monomer. The polymerization of AA is considered to be initiated from the transient radical as follows.

$$
(\mathrm{SCN})_{2}{ }^{-}+\mathrm{AA} \longrightarrow \mathrm{SCN}-\mathrm{AA} \cdot+\mathrm{SCN}^{-}
$$

Similarly, in the case of $\mathrm{KSeCN}$, an analogous transient radical was confirmed to be produced. However, the corresponding transient radical $\left(\mathrm{N}_{3} \cdot \text { or } \mathrm{N}_{6}{ }^{-}\right)^{21}$ for $\mathrm{N}_{3}{ }^{-}$could not be observed owing to no absorption in the visible region, although the $\mathrm{BA}$ triplet was quenched by $\mathrm{NaN}_{3}$. In any event, the initiation of polymerization with these transient radicals increased the number of initiating species and then the net polymerization rate of AA.

\section{Reaction Mechanisms}

From the results mentioned above, we can summarize some interesting features as follows.

1) BA played a role in sensitization for the polymerization of AA in aqueous solution, but $R_{\mathrm{p}}$ was relatively low and DP of the polymer obtained in the system was considerably small.

2) In the presence of $\mathrm{KSCN}, \mathrm{KSeCN}$, or $\mathrm{NaN}_{3}, R_{\mathrm{p}}$ increased with increasing concentration of the salt, but was held approximately constant in the higher concentration region where BA-phosphorescence was quenched completely. At the same time, DP of the polymer obtained also increased with $R_{\mathrm{p}}$.

3) In spite of no quenching of BAphosphorescence even in the high concentration of $\mathrm{Na}_{2} \mathrm{SeO}_{3}, R_{\mathrm{p}}$ was allowed to increase markedly with the salt concentration and DP decreased in a similar manner as the normal free radical polymerization.

4) In the absence of any salt, BA acted as a degradative chain-transfer agent at high concentration in the polymerization of $\mathrm{AA}$. However, the presence of a salt such as KSCN was found to remove the retarding effect of BA.

In its simple form, the process of a sensitized photopolymerization consists of four steps: light-absorption, initiation, propagation, and termination.

Light-absorption:

$$
\begin{aligned}
& \mathrm{BA} \stackrel{h v}{\longrightarrow}{ }^{1} \mathrm{BA} * \stackrel{\mathrm{ISC}}{\longrightarrow}{ }^{3} \mathrm{BA}^{*} \\
& { }^{3} \mathrm{BA} * \stackrel{k_{\mathrm{d}}}{\longrightarrow} 2 \mathrm{CH}_{3} \mathrm{CO} . \\
& { }^{3} \mathrm{BA}^{*} \stackrel{k_{\mathrm{e}}}{\longrightarrow} \mathrm{BA}+h v^{\prime}
\end{aligned}
$$




$$
{ }^{3} \mathrm{BA} * \stackrel{k_{\mathrm{nr}}}{\longrightarrow} \mathrm{BA}+\text { heat }
$$

The singlet excited state of $\mathrm{BA}$ is almost converted to the triplet state through intersystem crossing. The triplet state may decompose into an initiating radical species with a rate constant $k_{\mathrm{d}}$ and otherwise decay with emitting phosphorescence $\left(k_{\mathrm{e}}\right)$ or thermally without emitting $\left(k_{\mathrm{nr}}\right)$.

Initiation:

$$
\begin{array}{r}
\mathrm{CH}_{3} \mathrm{CO} \cdot+\mathrm{M} \stackrel{k_{\mathrm{i}}}{\longrightarrow} \mathrm{CH}_{3} \mathrm{CO}-\mathrm{M} \cdot \\
\left(=\mathrm{M}_{1} \cdot\right)
\end{array}
$$

Propagation:

$$
\mathrm{M}_{n} \cdot+\mathrm{M} \stackrel{k_{\mathrm{p}}}{\longrightarrow} \mathrm{M}_{n+1} .
$$

Termination:

$$
\mathrm{M}_{n} \cdot+\mathrm{M}_{m} \cdot \stackrel{k_{\mathrm{t}}}{\longrightarrow} \mathrm{P} \text { or } 2 \mathrm{P}
$$

where $\mathbf{M}, \mathbf{M}_{n} \cdot$, and $\mathrm{P}$ indicate monomer, growing polymer radical, and stable polymer, respectively, and $k_{\mathrm{i}}, k_{\mathrm{p}}$, and $k_{\mathrm{t}}$ are the rate constants of initiation, propagation, and termination, respectively.

In the Absence of Salt. Considering the features described above, the growing polymer radical might be destroyed by the interaction with the ground and/or excited state of BA, which would give a stable polymer and a hydrogen-transferred semidione radical $(\mathrm{BAH} \cdot)$. Here,

$$
\begin{array}{ccc}
\begin{array}{c}
\mathrm{BAH} \cdot: \\
\text { (semidione) }
\end{array} & \mathrm{CH}_{3}-\mathrm{C}-\dot{\mathrm{C}}-\mathrm{CH}_{3} \rightleftharpoons \\
& \stackrel{\mathrm{O} \text { OH }}{ } & \\
& & \mathrm{CH}_{3}-\underset{!}{\mathrm{C}}=\underset{\text { I }}{\mathrm{C}}-\mathrm{CH}_{3} \\
& & \cdot \mathrm{O} \quad \mathrm{OH}
\end{array}
$$

The ketyl-type structure in the resulting semidione radical seems to be very important and considerably stable ${ }^{22}$ leading to termination subsequently. So, the interaction can be referred to as degradative chain transfer as shown below.

Chain transfer:

$$
\mathrm{M}_{n} \cdot+\mathrm{BA} \stackrel{k_{\mathrm{r}}}{\longrightarrow} \mathrm{P}+\mathrm{BAH} \cdot
$$

$$
\begin{aligned}
& \mathrm{M}_{n} \cdot+{ }^{3} \mathrm{BA}^{*} \stackrel{k_{\mathrm{r}}{ }^{*}}{\longrightarrow} \mathrm{P}+\mathrm{BAH} \cdot \\
& \mathrm{M}_{n} \cdot+\mathrm{BAH} \cdot \stackrel{k_{\mathrm{td}}}{\longrightarrow} \mathrm{P}
\end{aligned}
$$

where $k_{\mathrm{r}}, k_{\mathrm{r}}^{*}$, and $k_{\mathrm{td}}$ are the rate constants for the retarding reactions by the ground and excited BA and for the termination by semidione radical, respectively.

Assuming a steady-state condition in the normal free radical polymerization without degradative chain transfer, $R_{\mathrm{p}}$ and DP are apparently expressed as well as eq 1 and 2 , respectively. However, if $\mathrm{BA}$ and/or ${ }^{3} \mathrm{BA}^{*}$ attack the growing polymer radical and bring about degradative chain transfer, the kinetics may be as follows.

$$
\begin{aligned}
& \begin{aligned}
\frac{\mathrm{d}\left[{ }^{3} \mathrm{BA}^{*}\right]}{\mathrm{d} t}= & 2.3 \phi \varepsilon \mathrm{d} I_{\mathrm{o}}[\mathrm{BA}] \\
-\left(k_{\mathrm{d}}+k_{\mathrm{e}}+k_{\mathrm{nr}}\right)\left[{ }^{3} \mathrm{BA}^{*}\right] & \\
- & k_{\mathrm{r}}^{*}\left[\mathrm{M}_{n} \cdot\right]\left[{ }^{3} \mathrm{BA}^{*}\right]=0
\end{aligned} \\
& \begin{aligned}
\frac{\mathrm{d}\left[\mathrm{CH}_{3} \mathrm{CO} \cdot\right]}{\mathrm{d} t}= & 2 k_{\mathrm{d}}\left[{ }^{3} \mathrm{BA}^{*}\right] \\
& -k_{\mathrm{i}}\left[\mathrm{CH}_{3} \mathrm{CO} \cdot\right][\mathrm{M}]=0
\end{aligned}
\end{aligned}
$$

$$
\begin{aligned}
\frac{\mathrm{d}\left[\mathrm{M}_{n} \cdot\right]}{\mathrm{d} t}= & k_{\mathrm{i}}\left[\mathrm{CH}_{3} \mathrm{CO} \cdot\right][\mathrm{M}]-k_{\mathrm{t}}\left[\mathrm{M}_{n} \cdot\right]^{2} \\
& -k_{\mathrm{r}}\left[\mathrm{M}_{n} \cdot\right][\mathrm{BA}]-k_{\mathrm{r}}^{*}\left[\mathrm{M}_{n} \cdot\right]\left[{ }^{3} \mathrm{BA}^{*}\right] \\
& -k_{\mathrm{td}}\left[\mathrm{M}_{n} \cdot\right][\mathrm{BAH} \cdot]=0
\end{aligned}
$$

From eq 12,

$$
\begin{aligned}
{\left[{ }^{3} \mathrm{BA}^{*}\right]=} & 2.3 \phi \varepsilon \mathrm{d} I_{0}[\mathrm{BA}] /\left(k_{\mathrm{d}}+k_{\mathrm{e}}\right. \\
& \left.k_{\mathrm{nr}}+k_{\mathrm{r}}{ }^{*}\left[\mathrm{M}_{n} \cdot\right]\right) .
\end{aligned}
$$

If most of ${ }^{3} \mathrm{BA}^{*}$ is consumed in eq 10 , the reaction may be regarded as a primary radical termination and $R_{\mathrm{p}}$ should show a zero-order dependence on the concentration of ${ }^{3} \mathrm{BA}^{*}$. As shown in Figure $1, R_{\mathrm{p}}$ is dependent on the half order of light intensity. Therefore, it can be assumed that $k_{\mathrm{d}}+k_{\mathrm{e}}+k_{\mathrm{nr}} \gg k_{\mathrm{r}}^{*}\left[\mathrm{M}_{n} \cdot\right]$ and then $\left[{ }^{3} \mathrm{BA}^{*}\right]=k[\mathrm{BA}] / k_{0}$, where $k=2.3 \phi \varepsilon \mathrm{d} I_{0}$ and $k_{0}=k_{\mathrm{d}}+k_{\mathrm{e}}+k_{\mathrm{nr}}$.

Furthermore, since the relation between 
$1 / \mathrm{DP}$ and $R_{\mathrm{p}}$ for various light intensities at $0.1 \mathrm{M} \mathrm{BA}$ was almost the same as that for various concentrations of BA at a fixed light, eq 10 might be much faster than eq 9 and immediately followed by eq 11 . Consequently, two glowing polymer radicals could be substantially terminated on eq 10 . Hence,

$$
\begin{aligned}
\frac{\mathrm{d}\left[\mathrm{M}_{n} \cdot\right]}{\mathrm{d} t}= & 2 k_{d}\left[{ }^{3} \mathrm{BA}^{*}\right]-k_{\mathrm{t}}\left[\mathrm{M}_{n} \cdot\right]^{2} \\
& -2 k_{\mathrm{r}}^{*}\left[\mathrm{M}_{n} \cdot\right]\left[{ }^{3} \mathrm{BA}^{*}\right]=0
\end{aligned}
$$

Although Deb $^{23}$ has proposed a method for analyzing the problems associated with primary radical termination and degradative chain transfer, the treatment is not properly applicable to our results owing to different experimental conditions. Hence, solving eq 15 as a quadratic equation, the stationary concentration of growing polymer radical $\left[\mathbf{M}_{n} \cdot\right]$ can be approximately expressed as follows,

$$
\left[\mathrm{M}_{n} \cdot\right]=\frac{-k C[\mathrm{BA}]+\left(k^{2} C^{2}[\mathrm{BA}]^{2}+2 k_{\mathrm{t}} k D[\mathrm{BA}]\right)^{1 / 2}}{k_{\mathrm{t}}}
$$

where $C=k_{\mathrm{r}}{ }^{*} / k_{0}$, and $D=k_{\mathrm{d}} / k_{0}$. $C$ is regarded as a measure of influence of degradative chain transfer and $D$ is the fraction of ${ }^{3} \mathrm{BA}^{*}$ decomposed in the decay processes.

As the overall rate of polymerization is expressed by eq 17 ,

$$
R_{\mathrm{p}}=k_{\mathrm{p}}\left[\mathrm{M}_{n} \cdot\right][\mathrm{M}]
$$

it is thought that if $C$ is zero or very small, $R_{\mathrm{p}}$ follows the proportional relation between $R_{\mathrm{p}}$ and $[\mathrm{BA}]^{1 / 2}$, and corresponds to that of the case involving no degradative chain transfer. As $C$ becomes large, the exponential dependence on [BA] may go down from $1 / 2$ toward zero order and finally $R_{\mathrm{p}}$ may approach zero. Therefore, at higher concentrations of $\mathrm{BA}, R_{\mathrm{p}}$ may be independent of concentration (Figure 5).

DP of the polymer obtained can be given as

$$
\mathrm{DP}=\frac{R_{\mathrm{p}}}{R_{\mathrm{t}}+R_{\mathrm{r}}^{*}}
$$

where $R_{\mathrm{t}}$ is the rate of production of polymer by the normal bimolecular termination and $R_{\mathrm{r}}^{*}$ is that by the degradative chain transfer. Then,

$$
\frac{1}{\mathrm{DP}}=\frac{k_{\mathrm{t}} R_{\mathrm{p}}}{k_{\mathrm{p}}^{2}[\mathrm{M}]^{2}}+\frac{2 k C[\mathrm{BA}]}{k_{\mathrm{p}}[\mathrm{M}]}
$$

In the absence of any salt, the second term of the right-hand side of eq 19 may be significant for large $C$ and then DP may be smaller than that of normal polymerization without such degradative chain transfer.

In the Presence of Salts. On the other hand, sufficient concentrations of $\mathrm{X}^{-}$(e.g., $\mathrm{SCN}^{-}$) to quench BA-phosphorescence can yield the initiating species $\mathrm{X}_{2}{ }^{-}$(e.g., $(\mathrm{SCN})_{2}{ }^{-}$).

$$
\begin{aligned}
& { }^{3} \mathrm{BA}^{*}+\mathrm{X}^{-} \stackrel{k_{\mathrm{q}}}{\longrightarrow} \mathrm{BA} \cdot \cdot^{-}+\mathrm{X} \cdot \\
& \mathrm{X} \cdot+\mathrm{X}^{-} \stackrel{K}{\rightleftharpoons} \mathrm{X}_{2} \cdot{ }^{-} \\
& \mathrm{X}_{2} \cdot{ }^{-}+\mathrm{M} \stackrel{k_{\mathrm{i}}^{\prime}}{\longrightarrow} \mathrm{X}-\mathrm{M} \cdot\left(=\mathrm{M}_{1} \cdot\right)+\mathrm{X}^{-}
\end{aligned}
$$

As the equilibrium constant $K$ is known generally to be large, $X$ - formed in eq 20 becomes immediately $\mathrm{X}_{2}{ }^{-}$, which initiates the polymerization instead of $\mathrm{CH}_{3} \mathrm{CO} \cdot$ resulting from direct breakage of ${ }^{3} \mathrm{BA}^{*}$. In a similar manner as before,

$$
\begin{gathered}
{\left[{ }^{3} \mathrm{BA}^{*}\right]=k[\mathrm{BA}] /\left(k_{0}+k_{\mathrm{q}}\left[\mathrm{X}^{-}\right]\right)} \\
\frac{\mathrm{d}\left[\mathrm{M}_{n} \cdot\right]}{\mathrm{d} t}=k_{\mathrm{q}}\left[{ }^{3} \mathrm{BA}^{*}\right]\left[\mathrm{X}^{-}\right]-k_{\mathrm{t}}\left[\mathrm{M}_{n} \cdot\right]^{2} \\
-2 k_{\mathrm{r}}^{*}\left[\mathrm{M}_{n} \cdot\right]\left[{ }^{3} \mathrm{BA}^{*}\right]=0
\end{gathered}
$$

Then, 


$$
\left[\mathrm{M}_{n} \cdot\right]=\frac{-k_{\mathrm{r}}^{*}\left[{ }^{3} \mathrm{BA}^{*}\right]+\left(k_{\mathrm{r}}^{* 2}\left[{ }^{3} \mathrm{BA}^{*}\right]^{2}+k_{\mathrm{t}} k_{\mathrm{q}}\left[{ }^{3} \mathrm{BA}^{*}\right]\left[\mathrm{X}^{-}\right]\right)^{1 / 2}}{k_{\mathrm{t}}}
$$

If the scavenging of growing radicals by ${ }^{3} \mathrm{BA} *$ could be neglected compared with the generation of $\mathrm{X}_{2} \cdot{ }^{-}$by eq 20 ,

$$
\begin{aligned}
R_{\mathrm{p}} & =k_{\mathrm{p}}\left[\mathrm{M}_{n} \cdot\right][\mathrm{M}] \\
& =k_{\mathrm{p}}\left(\frac{\left.k_{\mathrm{q}}{ }^{3} \mathrm{BA}^{*}\right]\left[\mathrm{X}^{-}\right]}{k_{\mathrm{t}}}\right)^{1 / 2}[\mathrm{M}] \\
& =k_{\mathrm{p}}\left(\frac{k[\mathrm{BA}]}{k_{\mathrm{t}}} \frac{k_{\mathrm{q}}\left[\mathrm{X}^{-}\right]}{\left(k_{0}+k_{\mathrm{q}}\left[\mathrm{X}^{-}\right]\right)}\right)^{1 / 2}[\mathrm{M}]
\end{aligned}
$$

Thus,

$$
\frac{1}{R_{\mathrm{p}}}=\frac{1}{k_{\mathrm{p}}[\mathrm{M}]}\left(\frac{k_{\mathrm{t}}}{k[\mathrm{BA}]}\right)^{1 / 2}\left(1+\frac{k_{0}}{k_{\mathrm{q}}\left[\mathrm{X}^{-}\right]}\right)^{1 / 2}
$$

Therefore, $R_{\mathrm{p}}$ should increase with the concentration of $\mathrm{X}^{-}$. This expectation has been well confirmed as shown in Figure 2.

In this case, DP may be expressed as follows from eq 18.

$$
\frac{1}{\mathrm{DP}}=\frac{k_{\mathrm{t}} R_{\mathrm{p}}}{k_{\mathrm{p}}^{2}[\mathrm{M}]^{2}}+\frac{2 k_{\mathrm{r}}^{*} k[\mathrm{BA}]}{k_{\mathrm{p}}[\mathrm{M}]\left(k_{0}+k_{\mathrm{q}}\left[\mathrm{X}^{-}\right]\right)}
$$

In the second term of the right-hand side in eq $27, k_{\mathrm{r}}^{*} /\left(k_{0}+k_{\mathrm{q}}\left[\mathrm{X}^{-}\right]\right)$corresponds to the ratio of the degradative transfer reaction to all decay processes of ${ }^{3} \mathrm{BA}^{*}$ and $\mathrm{DP}$ seems to increase with the concentration of $\mathrm{X}^{-}$. On the contrary, if the concentration of $\mathrm{X}^{-}$decreases to zero, eq 27 might coincide with eq 19 .

In the Case of $\mathrm{SeO}_{3}{ }^{2-}$. Although $\mathrm{SeO}_{3}{ }^{2-}$ has strong reducing power, it can not quench ${ }^{3} \mathrm{BA}^{*}$ directly. Therefore, the action of $\mathrm{SeO}_{3}{ }^{2-}$ seems to reduce semidone $(\mathrm{BAH} \cdot)$ yielding acetoin $\left(\mathrm{BAH}_{2}\right)$ and its own oxidized radical anion $\left(\mathrm{SeO}_{3} \cdot{ }^{-}\right)$. The resulting radical anion could reinitiate the polymerization smoothly.

$$
\begin{aligned}
& \mathrm{BAH} \cdot+\mathrm{H}^{+}+\mathrm{SeO}_{3}{ }^{2-} \stackrel{k_{\mathrm{red}}}{\longrightarrow} \\
& \mathrm{BAH}_{2}+\mathrm{SeO}_{3} \cdot- \\
& \mathrm{SeO}_{3} \cdot{ }^{-}+\mathrm{M} \stackrel{k_{\mathrm{rei}}}{\longrightarrow}-\mathrm{SeO}_{3}-\mathrm{M} \cdot\left(=\mathrm{M}_{1} \cdot\right)
\end{aligned}
$$

Then, the competition for BAH $\cdot$ between $\mathrm{M}_{n}$. and $\mathrm{SeO}_{3}{ }^{2-}$ would be essentially important.

Assuming a stationary state for $\mathrm{BAH} \cdot$, $\mathrm{SeO}_{3} \cdot{ }^{-}$, and $\mathrm{M}_{n} \cdot$ from eq 10,28 , and so on,

$$
\begin{aligned}
\frac{\mathrm{d}[\mathrm{BAH} \cdot]}{\mathrm{dt}}= & k_{\mathrm{r}}^{*}\left[\mathrm{M}_{n} \cdot\right]\left[{ }^{3} \mathrm{BA}^{*}\right] \\
& -k_{\mathrm{td}}[\mathrm{BAH} \cdot]\left[\mathrm{M}_{n} \cdot\right] \\
& -k_{\mathrm{red}}[\mathrm{BAH} \cdot]\left[\mathrm{SeO}_{3}{ }^{2-}\right]=0 \\
\frac{\mathrm{d}\left[\mathrm{M}_{n} \cdot\right]}{\mathrm{d} t}= & 2 k_{\mathrm{d}}\left[{ }^{3} \mathrm{BA}^{*}\right]-k_{\mathrm{t}}\left[\mathrm{M}_{n} \cdot\right]^{2} \\
& -2 k_{\mathrm{td}}[\mathrm{BAH} \cdot]\left[\mathrm{M}_{n} \cdot\right]=0
\end{aligned}
$$

But, since the kinetic solution of these equations for $\left[\mathrm{M}_{n} \cdot\right]$ is really very complicated, it is instructive to consider an extreme case in which sufficient $\mathrm{SeO}_{3}{ }^{2-}$ is added and an ideal chain transfer occurs eventually. Then, neglecting the third term in the middle of eq 31 and using the same abbreviations just as before,

$$
R_{\mathrm{p}}=k_{\mathrm{p}}\left[\mathrm{M}_{n} \cdot\right][\mathrm{M}]=k_{\mathrm{p}}\left(\frac{2 k_{\mathrm{d}} k[\mathrm{BA}]}{k_{\mathrm{t}} k_{0}}\right)^{1 / 2}[\mathrm{M}]
$$

Therefore, $R_{\mathrm{p}}$ would be greater than that in the absence of any salt. Further, eq 10 followed by eq 28 and 29 is regarded as an ideal chain transfer reaction $\left(k_{\mathrm{tr}}\right)$ between $\mathrm{M}_{n} \cdot$ and $\mathrm{SeO}_{3}{ }^{2-}$ yileding a stable polymer and a reinitiating radical species.

$$
\frac{1}{\mathrm{DP}}=\frac{k_{\mathrm{t}} R_{\mathrm{p}}}{k_{\mathrm{p}}{ }^{2}[\mathrm{M}]^{2}}+\frac{k_{\mathrm{tr}}\left[\mathrm{SeO}_{3}{ }^{2-}\right]}{k_{\mathrm{p}}[\mathrm{M}]}
$$

That is to say, DP may decrease with increasing concentration of $\mathrm{SeO}_{3}{ }^{2-}$.

In conclusion, such effects of inorganic salt additives on the BA-sensitized photopolymerization are specific to AA in aqueous solutions and were not found for any other watersoluble vinyl monomers such as methacrylamide, $N, N$-dimethylacrylamide, and methyl 
methacrylate tested. Therefore, such unique specificity to AA and BA needs to be investigated further.

\section{CONCLUDING REMARKS}

Although in aqueous solution 2,3-butanedione (biacetyl: BA) served as a photosensitizer for the polymerization of acrylamide (AA), BA itself acted as a degradative chain transfer agent and both $R_{\mathrm{p}}$ and DP of the resulting polymer were relatively low.

The addition of inorganic salts such as $\mathrm{KSCN}$ and $\mathrm{NaN}_{3}$ to the aqueous BAsensitized photopolymerization system increased not only $R_{\mathrm{p}}$ but also DP of the polymer obtained. These characteristic features were explained on the basis of the formation of initiating species with the interaction between the excited triplet state of BA and inorganic anions and the removal of influence of degradative chain transfer due to the excited BA. Moreover, the presence of $\mathrm{SeO}_{3}{ }^{2-}$, which has strong reducing power but no quenching ability toward the excited BA, could accelerate the photopolymerization of AA according to a normal kinetic scheme. These effects of inorganic salts on the photopolymerization were found only for AA, but not for any other vinyl monomers in aqueous solution.

\section{REFERENCES}

1. M. K. Mishra, J. Macromol. Sci., Rev. Macromol. Chem. Phys., C22, 409 (1982-83).

2. M. S. Matheson, E. E. Auer, E. B. Bevilacqua, and E. J. Hart, J. Am. Chem. Soc., 71, 497 (1949).
3. G. L. Osberg and D. J. Le Roy, Can. J. Chem., 29, 333 (1951).

4. G. P. Gladyshev and E. R. Rafikov, Vysokomol. Soyedin, 5, 700 (1963).

5. E. A. Lissi and M. V. Encina, J. Polym. Sci., Polym. Chem. Ed., 17, 2791 (1979).

6. M. V. Encina, J. Garrido, and E. A. Lissi, J. Polym. Sci., Polym. Chem. Ed., 23, 2481 (1985).

7. F. Takemura, Bull. Chem. Soc. Jpn., 35, 1073, 1078 (1962).

8. F. Takemura, M. Oomori, and H. Tanaka, Nippon Kagaku Zasshi, 89, 576 (1968).

9. F. Takemura, R. Matsuyama, K. Mori, and M. Nagai, Nippon Kagaku Zasshi, 92, 1102 (1971).

10. F. Takemura and E. Morita, Nippon Kagaku Zasshi, 92, 1107 (1971).

11. F. Takemura, E. Morita, and J. Asakura, Nippon Kagaku Kaishi, 376 (1973).

12. F. Takemura, K. Sakaguti, and Y. Tsuji, Nippon Kagaku Kaishi, 819 (1973).

13. F. Takemura and K. Sakaguti, Nippon Kagaku Kaishi, 1470 (1976).

14. K. Iwai, M. Uesugi, and F. Takemura, Polym. J., 17, 1005 (1985)

15. C. H. Bamford and C. F. H. Tipper, Ed., "Comprehensive Chemical Kinetics," Vol. 14A, "Free-radical Polymerization," Elsevier, Amsterdum, 1976, p 1.

16. J. Brandrup and E. H. Immergut, Ed., "Polymer Handbook," John-Wiley \& Sons, New York, N. Y., 1975, IV-9.

17. S. L. Murov, "Handbook of Photochemistry," Marcel Dekker, New York, N. Y., 1973, p 119.

18. P. Bortolus and S. Dellonete, J. Chem. Soc., Faraday Trans. 2, 71, 1338 (1975).

19. A. Treinin and E. Hayon, J. Am. Chem. Soc., 98, 3884 (1976).

20. A. Singh, A. R. Scott, and F. Sopchyshyn, J. Phys. Chem., 73, 2633 (1969).

21. M. S. Ram and D. M. Stanbury, J. Phys. Chem., 90, 3691 (1986).

22. E. T. Kaiser and L. Kevan, "Radical Ions," JohnWiley \& Sons, New York, N. Y., 1968, p 91.

23. P. C. Deb, Eur. Polym. J., 11, 31 (1975). 